\title{
LA INSTITUCIONALIZACIÓN DE LA FORMACIÓN DE LOS MAESTROS RURALES EM LA PROVINCIA DE ENTRE RÍOS (ARGENTINA, 1894-

\section{RESUMEN}

En este artículo se presenta una investigación sobre las políticas de formación para el magisterio rural desarrolladas en la provincia de Entre Ríos entre 1894 y 1914. Para ello, se examinan las instituciones creadas para tal fin, especialmente a partir de la reforma constitucional de 1903, es decir la Escuela Normal Rural provincial "Juan Bautista Alberdi” y los Cursos Temporarios para docentes en ejercicios y aspirantes. La articulación de ambas propuestas significó una forma de mejorar la preparación del magisterio rural y su jerarquización profesional, colaborando en el proceso de nacionalización de la población inmigrante radicada en la provincia, a través de la difusión de conocimientos y prácticas agropecuarias.

Palabras clave: Educación Rural, Escuela Normal Rural,Inmigración

1 Instituto Rosario de Investigaciones en Ciencias de la Educación (CONICET-UNR). Universidade Nacional de Entre Ríos (UNER) y Universidad Autónoma de Entre Ríos (UADER), Argentina. 


\section{A INSTITUCIONALIZAÇÃO DA FORMAÇÃO DOS PROFESSORES RURAIS NA PROVÍNCIA DE ENTRE RÍOS (ARGENTINA, 1894 -1914)}

\section{RESUMO}

Este artigo apresenta uma investigação sobre as políticas de formação de professores rurais desenvolvidas na província de Entre Ríos entre 1894 e 1914. Para isto, são examinadas as instituições criadas para tal fim, especialmente após a reforma constitucional de 1903, isto é, a Escola Normal Rural "Juan Bautista Alberdi" e os Cursos Temporários para professores em exercício e aspirantes. A articulação de ambas as propostas significou um caminho para melhorar a preparação dos professores rurais e sua hierarquia profissional, colaborando no processo de nacionalização da população imigrante radicada na província, através da disseminação de conhecimentos e práticas agrícolas.

Palavras chave: Educação Rural,Escola Normal Rural, Imigração.

\section{THE INSTITUTIONALIZATION OF THE TRAINING OF RURAL TEACHERS IN THE PROVINCE OF ENTRE RÍOS (ARGENTINA, 1894 -1914)}

\section{ABSTRACT}

This article presents an investigation about the training policies for rural teachers developed in the province of Entre Ríos between 1894 and 1914. Forthis purpose, the institutions created with this are examined, especially after the constitutional reform of 1903, that is to say the Provincial Rural Normal "Juan Bautista Alberdi" School and the Temporary Courses for teachers in exercises and aspirants. The articulation of both proposals meant a way to improve the preparation of rural for teachers atrork professional hierarchy, collaborating in the process of nationalization of the immigrant population settledin in the province, through the dissemination of knowledge and agricultural practices.

Keywords: rural education, rural normal school, immigration.

\section{L'INSTITUTIONNALISATION DE LA FORMATION DES ENSEIGNANTS RURAUX DE LA PROVINCE DE ENTRE RÍOS (ARGENTINE, 1894-1914)}

\section{RÉSUMÉ}

Cet article présente une enquête sur les politiques de formation des enseignants ruraux développées dans la province d'Entre Ríos entre 1894 et 1914. Pour cela, les institutions créées pour cet objectif sont examinées, spécialement à partir de la réforme constitutionnelle de 1903, c'est-à-dire l'École Normale Rurale provinciale "Juan Bautista Alberdi" et les Cours Temporaires pour enseignants actifs et aspirants enseignants. L'articulation des deux propositions a signifié une forme d'améliorer la préparation du magistère rural et de sa hiérarchisation professionnelle, en collaborant dans le processus de nationalisation de la population immigrante résident dans la province, à travers de la diffusion de connaissances et de pratiques agricoles. 
Revista História da Educação (Online), 2020, v. 24: e91406

Mots clefs : l'Education Rurale, École Normale Rurale, Immigration. 


\section{INTRODUCCIÓN}

Diferentes estudios históricos señalan que el proceso de profesionalización del magisterio inició su consolidación a fines del siglo XIX, a partir de la creación un subsistema de escuelas normales, necesario para la expansión de una escolarización primaria de alcance nacional (ALLIAUD, 1993; DIKER Y TERIGI, 1997; SCHUELER Y SOUTHWELL, 2011). Estos trabajos analizaron aspectos curriculares, pedagógicos y políticos de las escuelas normales ${ }^{2}$, aportando elementos básicos para la comprensión de la organización de la formación docente. No obstante, persistía una vacancia en el tratamiento específico de la preparación del magisterio rural, siendo este un tema que también registraba una demora en ser tratado en otros países de Latinoamérica con historiografías de la educación consolidadas (ASCOLANI, 2006). De tal modo, en la década pasada surgieron en forma casi paralela estudios específicos, y en cierto modo vinculados, sobre la formación de profesores rurales. Fue el caso de México, donde ha sido estudiado desde una perspectiva metodológica histórico-sociológica que procuró realizar una articulación entre las instituciones, las ideologías y las prácticas cotidianas (CIVERA, 2011). También del Brasil, donde se evidenció una orientación de la investigación que buscó captar la conexión entre la formación y la complejidad del funcionamiento escolar, atendiendo a las particularidades del medio rural (WERLE, 2008), y en la Argentina, donde los nuevos trabajos se centraron en la reconstrucción del desarrollo institucional de las escuelas normales rurales privilegiando los aspectos curriculares en un sentido amplio y observando el vínculo con las demandas sociales, así como con los desarrollos provinciales (ASCOLANI, 2007; GUTIÉRREZ, 2007). En todos los casos, el período central de estos estudios ha sido la primera mitad del siglo XX.

\footnotetext{
2 Véase Tedesco, 1986; Carli, 1993; Yannoulas, 1996; Puiggros, 2002; Lionetti, 2007; Firoucci,
} 2014. 
A partir de lo expuesto, en este artículo se analizan las políticas de formación para el magisterio rural en la provincia de Entre Ríos entre 1894 y 1914, sosteniendo la hipótesis de que la capacitación de los maestros entiéndase profesores primarios- rurales, a través de los Cursos Temporarios y la Escuela Normal Rural, impulsados por el Estado provincial durante esta etapa proponía una forma de intervención en las comunidades inmigrantes que se asentaban en el territorio entrerriano articulando los objetivos de nacionalización de la población y de mejoramiento de la producción agropecuaria. Para ello, en un primer apartado se examinan los proyectos y las iniciativas concretadas para resolver la falta de preparación de los maestros en el campo durante el período 1894 y 1903. En un segundo momento, se hace visible la propuesta de capacitación orientada a aspirantes y maestros en ejercicio, que se inicia en 1903, con los Cursos Temporarios, siendo este un tema sobre el cual no se registran estudios. En tercer lugar, se reconstruye la trayectoria institucional y los primeros años de funcionamiento de la Escuela Normal Rural, colocando especial atención en aspectos vinculados al curriculum y la organización interna. En el último punto se analizan las políticas educativas para el magisterio rural luego de la reforma constitucional de 1909, etapa que coincide con un momento de exacerbación del discurso nacionalista en diferentes ámbitos estatales (TERAN, 2008), especialmente el educativo.

La metodología usada en este artículo es esencialmente cualitativa y el enfoque historiográfico adoptado articula los aportes de varias orientaciones: la Historia social de las instituciones (ASCOLANI, 2007); el análisis de políticas públicas y Estado en escala subnacional (BOHOSLAVSKY y SOPRANO, 2010; PEREYRA, 2012); la tradición de la Historia regional, inspirada en la corriente de los Annales (AGUIRRE ROJAS, 2015), aunque también en desarrollos propios de la historiografía argentina (SOLIS CARNICER, 2015); y elementos de la Historia de la cultura escolar en la formación docente (GONÇALVES VIDAL, 2001).

La escritura de este trabajo utilizó un acervo documental diverso: 
expedientes escolares de la Dirección General de Enseñanza sobre el departamento Paraná, copiadores y correspondencia de la Escuela Normal Rural "Juan Bautista Alberdi"3, artículos de prensa de diferentes periódicos, leyes y decretos, memorias del Ministerio de Gobierno, boletines y memorias de la Dirección General de Enseñanza de la provincia de Entre Ríos.

\section{INMIGRACIÓN, COLONIZACIÓN AGRÍCOLA Y MAGISTERIO RURAL EN ENTRE RÍOS}

La provincia de Entre Ríos se ubica geográficamente en el litoral del país e integra el área productiva denominada región pampeana. Hasta mediados del siglo XIX, detentó un lugar de relevancia política en el plano nacional al convertirse en sede del gobierno de la Confederación Argentina. Durante esta etapa se instalaron las primeras colonias agrícolas con población inmigrante italiana, suiza valesana, francesa, entre otras, un movimiento que registraría mayor expansión a partir de la segunda mitad del siglo XIX (CARLI, 1993). Precisamente, luego de concluidas las últimas revueltas internas producidas a mediados de 1870 se produjo un importante crecimiento demográfico a causa del ingreso de población inmigrante. Entre las comunidades con mayor número de arribos destacaron los rusos-alemanes, en 1878, que se ubicaron inicialmente en el departamento Diamante. En 1892 llegó la comunidad israelita sostenidas por la Jewish Colonization Association que se instaló en los departamentos de Villaguay, Concepción del Uruguay y Colón.

La presencia de diferentes colectivos étnicos, especialmente en el medio rural, dispuestos a mantener sus tradiciones y lengua ubicó a la escuela común y obligatoria $^{4}$ en un lugar importante para lograr la nacionalización. El

\footnotetext{
3 Se agradece especialmente la colaboración brindada por las profesoras Mirta Nanni, Georgina Zarate y Sonia Unrein, personal responsable del Museo-Archivo "Nuestra Memoria" de la escuela Normal Rural “Juan Baustista Alberdi”.

4 En la Constitución Nacional de 1853 la educación primaria quedó bajo responsabilidad de los estados provinciales.
} 
establecimiento de escuelas en la "campaña" constituía un objetivo prioritario, pero estaba alejado de las posibilidades reales de la administración provincial porque se carecía de la infraestructura y el personal idóneo para la tarea. Se sumaban a esto, las características propias del medio rural que dificultaban el acceso y permanencia de los niños en los establecimientos escolares y la alternancia de los ciclos productivos que demandaban la presencia del grupo familiar para las tareas agrícolas. No obstante, las comunidades rurales creaban escuelas particulares que podían pertenecer a congregaciones religiosas, o pujaban por la concreción de escuelas fiscales.

Desde fines del siglo XIX se registraron algunos intentos para resolver el problema de la capacitación y falta de los maestros que afectaba, especialmente, al medio rural porque en el ámbito urbano se sostenía que las escuelas normales nacionales 5 proveían los maestros necesarios. En 1893 el Consejo General de Educación estableció una disposición que obligaba a los maestros que se desempeñaban en las escuelas comunes y que no tenían diploma a rendir un examen para su acceso. En esta norma se prescribían, además, las condiciones físicas, intelectuales y morales que debían tener los candidatos, dispensando de estas pruebas a quienes contaban con diplomas otorgados por las escuelas normales, por los Consejos Generales de Educación de las diferentes provincias, o antecedentes de un desempeño exitoso durante cuatro años de servicio. Los maestros con diplomas extranjeros debían revalidar sus títulos según la legislación vigente. Los resultados fueron escasos porque sólo cinco rindieron exámenes, dos registraron sus títulos y 17 obtuvieron diploma por la acreditación de buenos servicios. La percepción compartida por diferentes sectores sobre la preparación elemental de quienes se ocupaban de la enseñanza en la campaña demandaba una respuesta más eficaz. Así, la propuesta de fundar una escuela normal rural se convertía en prioridad para la administración provincial. Para fines de siglo, a las escuelas normales que creaba el Estado Nacional en distintos puntos del territorio se sumaban otras

\footnotetext{
5 En la ciudad de Paraná se fundó la primera escuela normal nacional en 1870. En Concepción del Uruguay funcionaba una institución análoga desde 1873.
} 
creaciones institucionales, como las escuelas normales regionales, de formación de preceptores y provinciales (ASCOLANI, 2007).

Aunque posteriormente la autoría del proyecto de creación de las escuelas normales rurales de la provincia estuvo sujeto a controversias que involucraban a figuras del normalismo local (DUPUY, 1913), esta propuesta venía circulando en diferentes ámbitos, como resultado de los viajes y el intercambio entre intelectuales. Una muestra de ello fue el informe que José Zubiaur ${ }^{6}$ elaboró tras asistir, como representante del país y de la provincia de Entre Ríos y Corrientes, al Congreso Pedagógico Internacional de Instrucción Primaria de Francia realizado en el marco de la Exposición Universal en 1889. En su presentación dedicó especial atención a la introducción de contenidos agrícolas en la escuela primaria francesa y la capacitación para su enseñanza de los maestros normales (ZUBIAUR, 1891), iniciativas que sugería debían replicarse localmente. En 1894, el profesor normal Leopoldo Herrera, presentó a la legislatura provincial un proyecto de creación de una escuela normal rural que contaba con el aval de otros colegas destacados como el presidente del Consejo General de Educación, Ernesto Bavio, y Alejandro Carbó. Esta escuela se ubicaría en el departamento Villaguay, punto central de la provincia. El programa de estudio comprendía dos años; en el primero se dictaría Pedagogía, Idioma Nacional, Historia Argentina, Nociones de Geografía General, Matemáticas, Ciencias Generales, Dibujo, Ejercicios Físicos y Música. En segundo año: Pedagogía, Idioma Nacional, Matemáticas, Instrucción Cívica, Geografía de la República Argentina, Nociones de Agricultura y Ganadería, Nociones de Química industrial y Música. La práctica de la enseñanza del curso normal se realizaría en las escuelas primarias de la localidad, para evitar la creación de una escuela de aplicación. La estructura de personal y presupuesto incluía un director y cuatro profesores. También, se proyectaba la creación de veinte becas para jóvenes pobres de los departamentos con la exigencia de

\footnotetext{
${ }^{6}$ Abogado y educador nació en Paraná, en 1851, y falleció en 1921. En 1886 fundó, junto a otros colegas, la revista "La Educación". Fue difusor de la introducción del trabajo manual en la escuela, la orientación agrícola de la educación primaria y la formación del magisterio rural.
} 
desempeño en las escuelas rurales el doble del tiempo de su usufructo, y se abría la posibilidad a que los municipios costearan algunas de ellas. La asignación presupuestaria-se decía-no afectaría a las rentas del Estado, ya que en un corto tiempo la escuela diplomaría maestros que ejercerían sus tareas en los distritos respectivos y se estipulaba que también estudiarían jóvenes sin beca y maestros sin título que estaban en ejercicio. El horizonte proyectado era ambicioso, al indicarse que en el término de 10 años reemplazarían todos los maestros sin título asentados en el campo y se imprimiría nueva orientación a la escuela primaria. Sin embargo, este proyecto no avanzó de su presentación en la legislatura. $\mathrm{Al}$ año siguiente, en 1895, el gobernador de la provincia indicaba que habían funcionado 355 escuelas atendidas por 700 maestros, cuya situación era la siguiente: “...Diplomados argentinos ( 58 varones y 128 mujeres) y extranjeros (63 varones y 137 mujeres). Sin diploma argentinos (76 varones y 221 mujeres) y extranjeros (196 varones y 304 mujeres)...” (MACIA, 1896, p. 49).

La mayor cantidad de maestros no diplomados se encontraba en el medio rural porque allí no se establecían los maestros egresados de las normales nacionales, que encontraban recepción inmediata en el ámbito urbano y, además, recibían una mejor remuneración. De este modo, en las escuelas rurales se empleaba personal sin titulación o maestros extranjeros “... ineptos con frecuencia para imprimir a la enseñanza espíritu nacional y patriótico que debe constituir su carácter fundamental...” (MACIA, 1895, p 29). En 1897, se modificó la Ley de Educación provincial y se estableció que la educación primaria debía ser "científica, regional y práctica". En consecuencia, se volvía insistir en la preparación del magisterio rural, acompañado de la creación de escuelas granjas que promovieran el desarrollo productivo. Una disposición relativamente novedosa para estimular la formación de maestros rurales fue el otorgamiento del título de competencia -mediante un año más de cursado- a los jóvenes que egresaban de la Escuela de Agricultura, Ganadería e Industrias Derivadas ubicada en Villa Urquiza, fundada en 1896. Esta resolución estaba presente desde que la escuela inició sus actividades y se registraron algunos egresos de maestros rurales que ocuparon cargos de directivos en escuelas de 
diferentes departamentos7.

En 1903 asumió la gobernación Enrique Carbó y durante su gestión, que se extendió hasta 1907, la administración provincial gozó de un período financiero favorable que posibilitó el dictado de algunas medidas para mejorar la situación productiva local. Le otorgó especial atención a la política de colonización agrícola con tierras fiscales, fundando seis nuevas colonias. También se atendió la situación de los asentamientos de las colectividades rusos-alemanes que, en 1903, se disponían a retornar a Rusia por las dificultades afrontadas desde su arribo a la provincia y el escaso rendimiento agrícola que tenían las colonias judías. Ante este panorama, el gobierno provincial dispuso la realización de una investigación sobre los factores "permanentes o accidentales" que habían influido en la producción de estas colonias. El primero de estos informes fue presentado en $1903 \mathrm{y}$, al año siguiente, el referido a las colonias de la Jewish. En ambos se describían las formas de vida de estas comunidades y se acentuaban representaciones peyorativas sobre las mismas concluyéndose, en líneas generales, que los colonos carecían de conocimientos y prácticas necesarias para la agricultura y presentaban diversos obstáculos para integrarse al país. Si bien este tipo de dictámenes sobre las comunidades inmigrantes no resultaba novedoso para la época, el tipo de estrategia que desplegó el Estado provincial para articular educación, trabajo agrícola y nacionalización merece atención.

Una medida clave para la comprensión de la política educativa del período fue la convocatoria a la Convención Constituyente para reformar la Constitución Provincial de 1883. La nueva Carta Magna se sancionó el 2 de junio de 1903 y conllevó cambios en la representación proporcional de diputados, las autonomías municipales y la justicia. En lo referido a educación, se estableció que la “... enseñanza pública podrá ser común y especial, la cual deberá referirse a las industrias agropecuarias, principalmente...” (CONSTITUCIÓN PROVINCIAL, 1903, p. 258) y se reorganizó el gobierno de la

7 AGER Fondo Educación. Serie XI Dpto. Paraná Caja No 171903 No Orden 22. 
educación. ${ }^{8}$ Las demandas previas sobre la necesidad de modificar los fines de la escuela común brindando una orientación práctica y el estimulo de la formación de personal idóneo para la agricultura y ganadería tuvieron rango constitucional. La responsabilidad del Estado provincial en el sostenimiento de la educación especial agropecuaria también incluía la formación de maestros normales rurales. En el artículo 256 de la Constitución se sostenía que "La enseñanza normal se contraerá a enseñar la teoría y la práctica profesional del magisterio para las escuelas rurales, estableciendo clases especiales en las escuelas primarias comunes más adecuadas y en las especiales de industrias rurales" (LEGISLACIÓN DE EDUCACIÓN PÚBLICA, 1904, p. 8). Todas las modificaciones fueron plasmadas en la Ley de Educación provincial $\mathrm{N}^{0} 1905$, sancionada en 1904. Otro aspecto central de la reforma lo constituyó la asignación presupuestaria destinada a educación, que no podía ser menor al 20\% de las Rentas Generales y para la educación especial se solicitó una subvención nacional a partir de 1906. La mejora de las finanzas públicas posibilitó abonar la deuda salarial de siete meses al magisterio y el aumento progresivo del presupuesto educativo garantizó una mayor inversión en infraestructura de las escuelas ubicadas en el medio rural, fundamentalmente. Durante toda la etapa la Dirección General de Enseñanza estuvo a cargo del profesor normal Manuel Antequeda,9 quien se desempeñó ininterrumpidamente entre 1903-1914.

\footnotetext{
${ }^{8}$ Se creó la Dirección General de Enseñanza con responsabilidad de la dirección técnica de las escuelas públicas y la superintendencia, inspección y vigilancia de enseñanza común y especial. Se deslindaban sus funciones de las correspondientes a Consejo de Administración.

9 Manuel Antequeda nació en Mendoza en 1860 y falleció en 1920. Destacado Profesor Normal Nacional, ejerció cargos en Buenos Aires, Mendoza y San Juan. En 1903 fue convocado para presidir la Dirección General de Escuelas en Entre Ríos.
} 


\section{¿UNA MEDIDA TRANSITORIA? LOS CURSOS TEMPORARIOS PARA MAESTROS RURALES}

En 1903 los datos estadísticos sobre el magisterio provincial presentados por las dependencias oficiales mostraban que alrededor del 65\% de los maestros en ejercicio carecían de diploma, un problema que se agravaba en las escuelas particulares. Atento a esta situación, una de las primeras medidas que adoptó Manuel Antequeda fue la firma, el 2 de abril, del decreto que creaba los Cursos Temporarios para maestros en ejercicio que no tuviesen titulación o aspirantes, y la organización de Conferencias Pedagógicas para maestros del ámbito urbano y rural ${ }^{10}$. Los cursos temporales fueron concebidos como una política breve que se desmantelaría cuando las escuelas normales rurales- que se proyectaban construir -brindaran los egresados necesarios.

La apertura de los Cursos Temporarios para el verano de 1903-1904 derogaba las disposiciones previas sobre expedición de diplomas, exámenes y otorgamiento de títulos. En este primer ensayo funcionaron en las ciudades cabeceras de los departamentos Concepción del Uruguay, Victoria, Gualeguay, Paraná, Gualeguaychú y Concordia; ${ }^{11}$ sin una distribución y secuenciación precisa, en los cursos se dictaron las siguientes materias: Pedagogía, Instrucción Moral y Cívica, Historia, Geografía, Aritmética, Idioma Nacional, Ganadería, Agricultura y Práctica de la Enseñanza. A cargo de las cátedras se designaron autoridades y personal docente habilitado que, su mayoría, provenía de las escuelas normales o colegios nacionales que funcionaban allí. Se registró una importante cantidad de inscriptos, ya sea maestros en ejercicio o aspirantes a serlo. Del total de 311 inscriptos, 124 eran maestros fiscales, 68 particulares y 119 aspirantes, asistiendo la mayoría. Estos datos de inscripción y asistencia resultaban, para la Inspección General, una prueba del éxito alcanzado y contrastaban con las opiniones pesimistas expresados en la prensa local. Los resultados de los exámenes de los aspirantes arrojaron 78 aprobados, que

\footnotetext{
${ }^{10} \mathrm{El} 27$ de octubre de 1903 se fijaron los requisitos para la inscripción a los Cursos Temporarios. ${ }^{11}$ Este Curso no se completó por falta de profesor de la materia de agricultura y ganadería.
} 
obtuvieron el título de maestro rural provincial. Quienes resultaron aplazados debían volver a cursar el curso completo y aquellos que habían aprobado la mitad de materias podían terminarlo el año próximo. Los datos de este primer curso también mostraron la importante presencia femenina en el magisterio rural, ${ }^{12}$ pese a que desde los ámbitos oficiales se promovió la formación de maestros varones por la supuesta idoneidad de los mismos en el gobierno escolar y en el medio que se desempeñarían.

En noviembre de 1904, se reglamentó el funcionamiento de los Cursos Temporarios, se redujo la cantidad de sedes por la apertura reciente de la Escuela Normal Rural y se priorizó la ubicación por razones estratégicas, quedando Paraná, Uruguay, Rosario del Tala y Concordia (Figura I). Esta reglamentación producida a partir de la experiencia práctica anterior apuntaba a mejorar el notable desequilibro de preparación que habían arrojado los exámenes del período anterior:

(...)si con el primer ensayo pudo explicarse la liberalidad con que se abrió la puerta a todos, ahora en momento que funciona una Escuela Normal especial no hay razón y hay conveniencia en seleccionar y admitir solamente a los que ofrezcan garantías de buenos resultados y no sean obstáculos para la marcha regular de los Cursos... (AGER, FONDO EDUCACIÓN. SERIE DEPARTAMENTO PARANÁ 19021907).

Figura I. Mapa con la ubicación de Escuelas Agropecuarias, Normal Rural y los Cursos Temporarios en 1904

\footnotetext{
${ }^{12}$ Los aprobados por departamento: Uruguay 23 mujeres y 13 varones; Gualeguay cinco mujeres y cuatro varones; Victoria cinco mujeres y seis varones; Gualeguaychú siete mujeres y seis varones y Paraná con cinco mujeres y cuatro varones.
} 


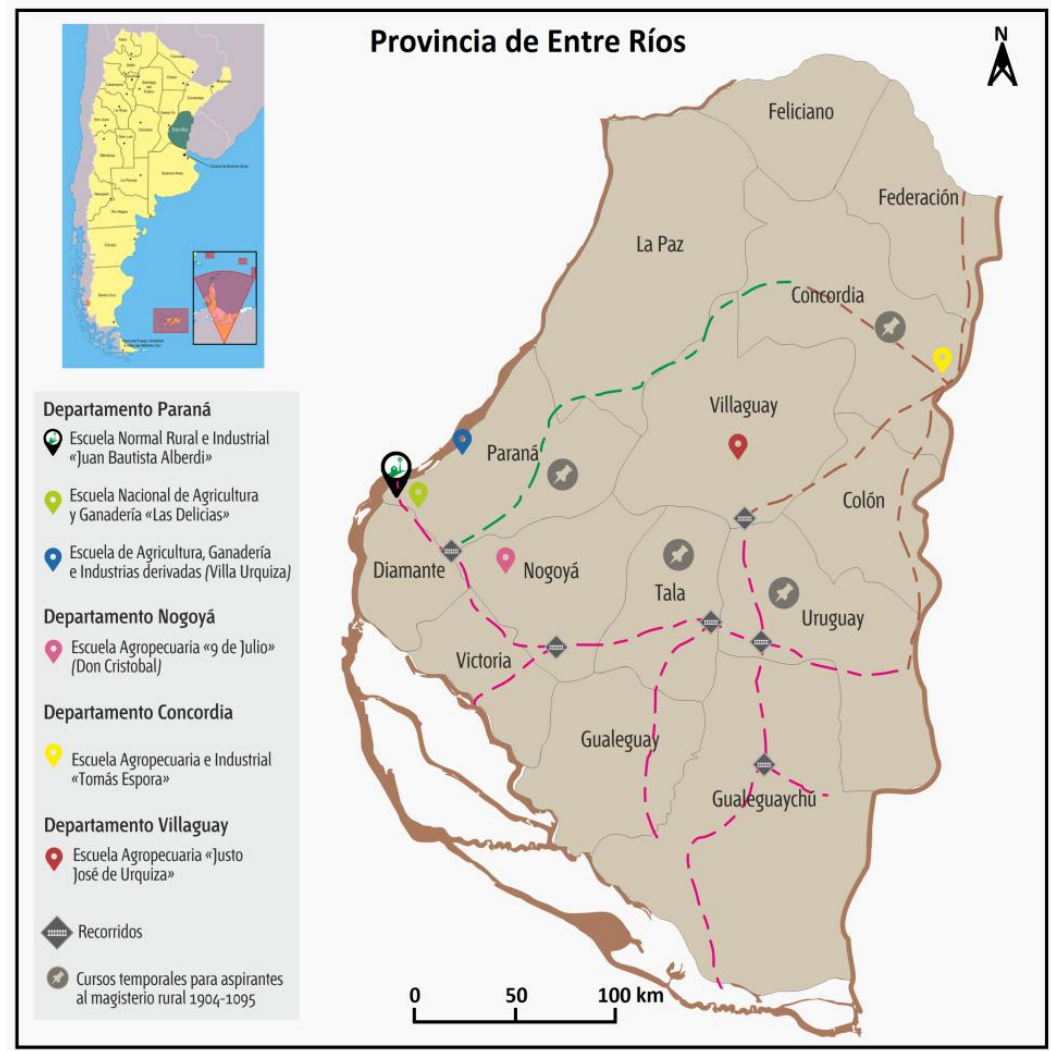

Fuente: Elaboración propia tomando referencias de la Memoria del Ministerio de Gobierno. Año económico 1899. Paraná, Tipografía y encuadernación EL Paraná, 1900.

Se exigía comprobar el ejercicio previo del magisterio o haber concurrido a los cursos del año anterior; en caso de no tener antecedentes docentes debían rendir exámenes de ingreso y tener una edad mínima de 17 años para las mujeres y 18 los varones. Para quienes se desempeñaban como maestros, la inasistencia a los cursos sin justificación conllevaba la cesantía en el cargo. Asimismo, se establecía un sobresueldo para los maestros que tenían a cargo su familia, por los gastos que implicaba el desplazamiento hasta la ciudad donde se dictaba el curso. La composición del personal y el dictado de las materias estaba organizado por un director responsable de las cátedras de Pedagogía, Práctica de la Enseñanza e Instrucción Cívica con ocho horas. Las cátedras de Aritmética, Idioma Nacional y Caligrafía tenían siete horas y las de Geografía, Historia y Moral, con cinco horas. Completaban este cuadro el profesor a cargo del curso de Agricultura y Ganadería con cuatro horas y una 
maestra para la Escuela de Aplicación. Los exámenes de los cursos se tomaban a mediados de febrero y cada profesor tenía bajo su responsabilidad la evaluación de los aspirantes según el formato tradicional de las "bolillas", a excepción de Idioma Nacional.

Al año siguiente, el 24 de noviembre de 1905, se suprimieron por resolución los Cursos Temporarios para Maestros no diplomados y Aspirantes “... con los fines con que se estableció por resolución de fecha 27 de octubre de 1903." (BOLETÍN DE EDUCACIÓN, 1905) El cierre de los cursos temporales en las sedes departamentales habilitaba la posibilidad de completar las materias pendientes en las mesas examinadoras que se abrirían en febrero en distintos puntos de la provincia. Aunque se suprimía la modalidad del primer Curso Temporario descentralizado territorialmente, se reafirmaba la necesidad de capacitar a los maestros que dictaban clases en escuelas rurales con chacras anexas. Para ellos se dispuso, en la misma resolución, la creación de un Curso Especial de Agricultura Práctica para varones y de carácter obligatorio que funcionaría entre diciembre y febrero en la Escuela Normal Rural o la posibilidad de rendir exámenes según los planes vigentes en dicha escuela durante los plazos dispuestos y a cargo del mismo personal responsable del curso normal. Por medio de esta resolución se institucionalizó esta capacitación de verano para varones en la Escuela Normal Rural, quedando sin posibilidades de cursar la población femenina que solo podía rendir en las mesas examinadoras habilitadas. A fines de 1906, se informaba que los Cursos Temporarios habían otorgado titulación a unos 200 maestros.

En 1908 se establecieron modificaciones al Reglamento de la Enseñanza Común vigente que adaptaba la normativa escolar "al funcionamiento concreto de las escuelas" e incorporaba las reformas que se venían introduciendo en la legislación educativa provincial. En el apartado referido al magisterio se definían las condiciones para su ejercicio y se establecía que los Comisionados Seccionales iban a abrir en sus oficinas el registro de inscripción para exámenes de maestros y aspirantes entre enero y febrero de 
cada año. Se mantenían los mismos requisitos exigidos con anterioridad en edad, condiciones de salud y moralidad. Para los aspirantes al título de maestro rural debían rendir el Curso siguiendo los programas sintéticos de la Escuela Normal Rural, sin modificaciones. Las mujeres, en cambio, los contenidos de agricultura se limitaban a nociones elementales de horticultura y jardinería, organización de huerta escolar y jardín escolar, preparación de la fiesta del árbol; en zootecnia nociones elementales sobre avicultura y en industrias rurales contenidos elementales de lechería, fabricación manteca, queso e industria sericícola. Se flexibilizaban criterios de evaluación al considerarse validas las pruebas de los aprobados en las dos terceras partes de las materias rendidas, pero si en las próximas vacaciones no aprobaban perdían validez. El título de maestro rural estaba ubicado en el último lugar de la jerarquía de titulaciones docentes, aunque habilitaba para ascender en la carrera del magisterio.

\section{ENTRE LA NOVEDAD Y LA NECESIDAD: LA CREACIÓN DE UNA ESCUELA NORMAL RURAL E INDUSTRIAL}

La creación de esta institución sobresale como una de los aspectos más importantes de la gestión de Manuel Antequeda, pero su fundación no fue un acto aislado. En la Constitución Provincial de 1903 se impulsó la educación agropecuaria por medio de la creación de escuelas con esta finalidad en los distintos departamentos ${ }^{13} \mathrm{y}$ también a través de la enseñanza normal rural. Por decreto se instituyó la fundación de dos escuelas normales rurales e industriales que iban a instalarse en jurisdicciones a definir. Para esto, el gobierno envió un

13 Originalmente, se pretendía organizar una escuela agropecuaria en cada uno de los 14 departamentos de la provincia. Finalmente, sólo se fundaron: la escuela agropecuaria e industrial "Tomás Espora" (1904) en Concordia, la agropecuaria "9 de julio" (1904) en Nogoyá, Agropecuaria "Justo José de Urquiza" (1905) en Villaguay. Completaba este cuadro la escuela Nacional de Agricultura y Ganadería "Las Delicias" (1900) y la escuela agropecuaria de Villa Urquiza (1896). 
técnico encargado de estudiar las condiciones óptimas para su emplazamiento. El proyecto inicial presentado por el senador Medrano tenía como objetivo crear:

(...) una escuela especial para la enseñanza de las industrias agropecuarias y formación de maestros rurales con asiento en el establecimiento fiscal de Algarrobitos (...) para ampliar la educación común despojándola de su espíritu teórico y literario (...) para imprimirle tendencias prácticas, marcadamente productoras e industriales (CÁMARA DE SENADORES, DIARIO DE SESIONES, $13^{\circ}$ SESIÓN ORDINARIA, 25 de JULIO DE 1903, p 38- 39).

Finalmente por Ley $\mathrm{N}^{0} 1897$, del 20 enero de 1904, se aprobó la adquisición de 3. 708 hectáreas en el paraje Tezanos Pinto, departamento Diamante, y se autorizó al Poder Ejecutivo provincial para enajenar la propiedad adquirida -que comprendía la ex estancia del gobernador Fabre- para la ubicación de la Escuela Normal Rural. El 19 de marzo de 1904, se trató el pedido de la Dirección General de Enseñanza donde solicitaba al PE que le transfiera una fracción de 400 hectáreas para el establecimiento de una escuela "agropecuaria y normal para maestros de campaña" cuya tarea era la promoción de la agricultura y ganadería local. Se esperaba que los alumnos:

(...).aprendan la teoría y la práctica del arte de enseñar y adquieran conocimientos especiales en los siguiente: en la ejecución de las diferentes operaciones del cultivo; trabajo del suelo, siembra; recolección cuidado de cosecha, conservación y utilización de productos, etc.; en el manejo y montaje de la maquina y útiles agrícolas; en la cría, alimentación, engorde, cuidado del ganado, etc.; en los trabajos relativos a la transformación industrial de los productos agropecuarios; en las cuestiones referentes a las enfermedades, plagas, etc., que perjudican a la agricultura y ganadería.. (EL TRIBUNO, $1904 \mathrm{~s} / \mathrm{p}$ ).

La Escuela14 inició sus actividades el 17 de julio de 1904 y la duración de

${ }^{14} \mathrm{El} 10$ de noviembre de 1904 se estableció por decreto que esta institución se denominará Juan 
los estudios comprendía tres años -uno menos que las normales nacionalesdivididos en semestres. Las actividades escolares se distribuían entre mayo y noviembre, con vacaciones de quince días después de los exámenes finales de cada semestre, aunque este calendario se modificaba de acuerdo a las necesidades productivas del establecimiento. Las condiciones de ingreso no diferían de otras instituciones de este tipo: los alumnos varones debían tener una edad que oscilara entre los 16 y 21 años, no padecer defectos físicos, tener costumbres acordes a la moral y haber aprobado el $5^{0}$ grado o, en su defecto, rendir un examen de ingreso. Los aspirantes podían ser pensionados o tener becas y para acceder a ésta última se debía probar mediante autoridad competente la condición de pobreza. Este requisito aporta elementos sobre las características socioeconómicas de los aspirantes al magisterio rural, en su mayoría carente de recursos económicos propios para costear sus estudios. El usufructo de la beca obligaba al desempeño por tres años en el ámbito rural, con lo cual se aseguraba la devolución del monto recibido y en caso de no aprobar las materias correspondientes a cada año se perdía el beneficio. Se establecieron 28 becas, previendo dos alumnos becados por cada departamento, que no pudo concretarse por falta de espacio físico. Las actividades se iniciaron con 20 alumnos, algunos provenientes de la escuela de Agricultura, Ganadería e Industrias Derivadas emplazada en Villa Urquiza.

El interés de formar un mínimo de maestros por cada departamento provincial y los problemas de concurrencia que generaba puede explicar, en parte, la preferencia por la instalación de un internado sólo para varones. Otro punto a considerar respecto al acento en la formación de maestros varones lo podría constituir la progresiva feminización de la población de la Normal Nacional de Paraná que, a partir de 1895, ya superaba el 50\% de la población total del curso (YANNOULAS, 1996). Sin embargo, para los funcionarios el argumento principal obedecía a fines prácticos, al no contarse con infraestructura necesaria para albergar a las jóvenes en la escuela ni disponer de

Bautista Alberdi, para “...honrar la memoria de uno de los patricios que con más interés se ocupara del género de estudios”. 
una fluida red de transporte que facilitara el recorrido diario de los veinte kilómetros que separaban la ciudad de Paraná de Tezanos Pinto.

En el Reglamento Interno, prescripto casi en simultáneo con la apertura de la escuela, se regulaban las funciones y responsabilidades del personal directivo, profesores, alumnos-maestros, encargados de las diferentes secciones, la disciplina y los exámenes. Se establecía una estructura organizacional y presupuestaria bastante más compleja que las escuelas agropecuarias, componiéndose de un director ${ }^{15}$ que debía tener título de profesor normal y un vicedirector -con título de ingeniero agrónomo- con funciones de jefe de cultivos. El organigrama institucional se completaba con un secretario contador, tres profesores, un maestro normal, un maestro quesero cremero, un capataz arboricultor, un agricultor, un oficial carpintero, un oficial herrero, un ecónomo, un cocinero, una lavandera, un sirviente (Tabla I). El dictado teórico de las materias se realizaba por la mañana y los trabajos prácticos por la tarde, sin detallarse con especificidad cuantas horas se le dedicaba al trabajo en las secciones, situación que motivó diferentes conflictos con los alumnos por la cantidad de trabajo demandada. Se contaba con escuela elemental anexa donde se realizaban las prácticas de los alumnos-maestros y recibían niños de las zonas aledañas.

\footnotetext{
15Entre 1904 y 1908 alternaron en el cargo ocho directivos (Ramón Medrano, Francisco Fernández, Luis Goncebatt, Manuel Martínez, Modesto Quiroga, Felipe Gardell, Gervasio Barzola y Soriano Romero). A fines de 1908 asumió Amadeo Autcher, funcionario que se mantuvo en el cargo hasta 1912.
} 
Tabla 1. Presupuesto para la escuela Juan Bautista Alberdi (1905) ${ }^{1}$

\begin{tabular}{|c|c|c|}
\hline \multirow{2}{*}{ PERSONAL } & \multicolumn{2}{|c|}{ ESTIPENDIO } \\
\hline & MENSUAL & ANUAL \\
\hline Director y Profesor & 300 & 3600 \\
\hline Vice Director Ingeniero Agrónomo & 200 & 2400 \\
\hline Profesor & $120(\mathrm{n}=3)$ & 4320 \\
\hline Maestro Normal & 100 & 1200 \\
\hline Secretario contador tesorero & 60 & 720 \\
\hline Maestro quesero-cremero & 90 & 1080 \\
\hline Capataz Arboricultor & 90 & 1080 \\
\hline Agricultor & 90 & 1080 \\
\hline Oficial carpintero & 80 & 960 \\
\hline Oficial herrero & 80 & 960 \\
\hline Beca para alumno-maestro & $12(n=28)$ & 4032 \\
\hline Mantenimiento por alumno & $20(n=28)$ & 6720 \\
\hline Ecónomo & 90 & 1080 \\
\hline Cocinero & 35 & 420 \\
\hline Lavandera & 40 & 480 \\
\hline Sirviente & 30 & 360 \\
\hline \multicolumn{3}{|l|}{ GASTOS DE FUNCIONAMIENTO } \\
\hline Manutención del Personal & 100 & 1200 \\
\hline Gastos de instalación, peones y herramientas & 500 & 6000 \\
\hline Total & 2541 & 37.692 \\
\hline
\end{tabular}

Fuente: Ley de presupuesto general de la administración,1905. (n= número de individuos).

La organización de los estudios y las prácticas agrícolas, ganaderas y de las industrias derivadas de estas actividades representaba los aspectos novedosos del plan de formación de maestros rurales que debían egresar con conocimientos para el sostenimiento y uso racional de una chacra. El primer plan de estudio tenía las siguientes materias: 
Tabla 2. Plan de estudio de la escuela Juan Bautista Alberdi (1904) ${ }^{1}$

\begin{tabular}{|c|c|c|c|}
\hline \multirow{2}{*}{ MATERIAS } & \multicolumn{3}{|c|}{ AÑ̃ } \\
\hline & $1^{0}$ & $2^{0}$ & $3^{0}$ \\
\hline Pedagogía & $\checkmark$ & $\checkmark$ & $\checkmark$ \\
\hline Aritmética & $\checkmark$ & $\checkmark$ & $\checkmark$ \\
\hline Castellano & $\checkmark$ & $\checkmark$ & $\checkmark$ \\
\hline Historia & $\checkmark$ & $\checkmark$ & $\checkmark$ \\
\hline Geografía & $\checkmark$ & $\checkmark$ & $\checkmark$ \\
\hline Agricultura general & $\checkmark$ & $\checkmark$ & - \\
\hline Geometría y Dibujo & $\checkmark$ & $\checkmark$ & $\checkmark$ \\
\hline Industrias Rurales & - & $\checkmark$ & - \\
\hline Zootecnia general & $\checkmark$ & - & - \\
\hline Ganadería & - & $\checkmark$ & $\checkmark$ \\
\hline Moral e Instrucción cívica & - & - & $\checkmark$ \\
\hline Agricultura especial & - & - & $\checkmark$ \\
\hline Ejercicios prácticos de pedagogía y agrícolas & $\checkmark$ & $\checkmark$ & $\checkmark$ \\
\hline
\end{tabular}

Fuente: Legislación educativa 1904.

En las materias agropecuarias los contenidos que se desarrollaban ofrecían conocimientos técnicos sobre los procesos agrícolas, ganaderos y la industrialización de sus derivados (lechería, cremería, sericultura, apicultura, vitivinicultura). Varios conflictos acaecidos en esta etapa inicial permiten comprender las formas de apropiación de la propuesta curricular a nivel institucional, como también en un plano más amplio entender que la preparación del magisterio y el desarrollo de una institución con tendencia a la experimentación agropecuaria resultaba difícil de comprender para algunos sectores que cuestionaban el carácter oneroso de la misma (CIAN, 2018). A fines de 1907 concluyeron sus estudios los primeros 17 maestros normales rurales. Fueron destinados, en la misma resolución de egreso, a las escuelas elementales con anexos agropecuarios incluidas en la Ley de Presupuesto de 1908. 


\section{LA FORMACIÓN DEL MAGISTERIO RURAL EN LA REFORMA CONSTITUCIONAL DE 1909}

Las elevadas tasas de analfabetismo de la población constituían una de las problemáticas centrales para la administración educativa nacional y provincial. Dichos indicadores variaban permanentemente, en parte, por el arribo de población inmigrante que durante este período registró su principal expansión. En el caso de Entre Ríos, los datos del segundo Censo Escolar Nacional, de 1909, arrojaron altas tasas de analfabetismo y semialfabetismo. ${ }^{16}$ Estos datos generaron malestar en la administración provincial que mandó a realizar su propio censo en 1910 para contrarrestar la información arrojada por la dependencia nacional. Con una población mayoritariamente ubicada en el ámbito rural, los esfuerzos del Estado provincial se concentraron, a partir de 1903, en el mejoramiento de la escuela rural que tenía una duración menor a la escolaridad exigida por ley, ya que en su mayoría sólo se dictaba hasta tercer grado. De esta forma, mejorar la infraestructura, aumentar el número de escuelas, asegurar la asistencia de la población y preparar los maestros para el ejercicio en el medio rural constituyeron algunos de los componentes de la política educativa encarada a partir de esta etapa. Un punto de articulación de estas disposiciones lo representó el objetivo de difusión de conocimientos y prácticas agropecuarias que transformarían el panorama productivo de la provincia, fortaleciendo la agricultura cerealera y los cultivos regionales.

Casi inmediatamente a la puesta en funcionamiento de los lineamientos de esta política educativa, diversos sectores de la sociedad civil y del oficialismo gobernante cuestionaban estos objetivos por el elevado presupuesto que insumía. Uno de los puntos de mayor controversia lo constituía la organización de la Escuela Normal Rural que, en 1908, demandaba un presupuesto que cuadruplicaba a las escuelas agropecuarias y brindaba un reducido número de egresados. Desde la prensa se hacía hincapié en la necesidad de cerrar o

\footnotetext{
${ }^{16}$ Los datos del segundo censo escolar arrojaban 39, 5\% analfabetos, 9,3\% de semi alfabetos y
} $51,2 \%$ de alfabetos en la provincia de Entre Ríos. 
nacionalizar las escuelas especiales y emplear ese dinero en la expansión de la alfabetización y nacionalización de la población inmigrante, donde se encontraban comunidades que ofrecían resistencias a la integración ciudadana propuesta por el modelo liberal conservador.

La preocupación por la nacionalización se expresó con mayor fuerza en 1908, cuando se produjo el conflicto entre el Director General de Enseñanza, Manuel Antequeda, y el Inspector Nacional de escuelas de provincia del Consejo Nacional de Educación, ${ }^{17}$ Ernesto Bavio, por el tipo de educación que brindaban las escuelas de las comunidades ruso- alemanes y judías (LÓPEZ, 1999). Pero en esta instancia, la proyección de este suceso se exacerbó producto de la acentuación del discurso nacionalista en diferentes ámbitos, entre ellos el Consejo Nacional de Educación, presidido por José Ramos Mejía. El trasfondo del enfrentamiento entre funcionarios mostraba la disputa por la ubicación de las escuelas primarias rurales nacionales creadas por la Ley No 4874 en 1905. La instalación de estas instituciones tenía como fin ayudar a las provincias a asegurar la instrucción elemental de la población, especialmente en el medio rural, pero en la práctica aún no habían tenido el resultado esperado, con lo cual el problema del analfabetismo y la nacionalización de la población seguía irresuelto.

Estos precedentes incidieron en la introducción de cambios en el apartado sobre educación de la Constitución Provincial reformada en 1909, dado que se modificaron aspectos vinculados a la Educación Común -junto a otros como el Superior Tribunal de Justica, Concejo Deliberante y Disposiciones Transitorias-. En lo referido a educación, la comisión resolvió que la Educación Común “... comprenderá exclusivamente la instrucción primaria, será gratuita, obligatoria y de carácter esencialmente nacional...” y se restableció el gobierno de la educación bajo la dirección del Consejo General de Educación. ${ }^{18}$ De esta

\footnotetext{
${ }_{17}$ Este órgano tenía jurisdicción- según la Ley No 1420-sobre las escuelas de la Capital Federal, Colonias y Territorios Nacionales. A partir de la Ley $\mathrm{N}^{0} 4874$ extendió su acción a las provincias por medio de la creación de escuelas primarias.

${ }^{18}$ Se vuelve a la estructura del Consejo General de educación bajo la dependencia del Ministerio
} 
forma se sostenía que las políticas educativas provinciales debían tener como objetivo central atender a la educación primaria, disponiendo esta área de los recursos que se dedicaban a la enseñanza pública y, en consecuencia, reducir el financiamiento que hasta la fecha se había brindado a la educación agropecuaria. La Ley de Educación de 1909 plasmó estas disposiciones y reforzó mediante diversas prácticas escolares, como las conmemoraciones y el homenaje a los símbolos patrios, el objetivo de nacionalización.

Los cambios constitucionales y su correlato en la legislación educativa no afectaron los aspectos centrales de las disposiciones en vigencia sobre la capacitación de los maestros rurales. Con esto se alude al Curso de Agricultura Práctica, las Mesas examinadoras, la revalidación de los títulos de maestros extranjeros y el otorgamiento de un certificado habilitante para los maestros sin título que se desempeñaban en la provincia, previa constatación de su trabajo. No obstante, se dio un nuevo impulso a los Cursos Temporarios, que volvían a dictarse con la estructura curricular previa, aunque la única sede para cursar estaba en la Escuela "Alberdi" y se colocaba especial atención en formar maestros de las colonias, puntualmente, rusas y judías. Esto se materializó en el otorgamiento de cuatro lugares para maestros argentinos de escuelas rusas e israelitas en los cursos temporarios de 1909-1910 ${ }^{19}$. Desde la prensa local se publicitaba los logros que implicaba porque:

(...) están mezclados jóvenes y viejos, argentinos y extranjeros; siendo en su mayor numero israelitas y rusos alemanes (...) Se trabajará en la chacra tanto o más que se teorizará y los mismos estudiantes han llegado en oportunidad para levantar la no despreciable cosecha de la escuela y adquirir ciertos conocimientos que se aprenden en las aulas.(...) Los demás alumnos extranjeros proviene de las escuelas, rusos alemanes, del departamento Diamante (...)Es digno de notarse que los maestros judíos que están recibiendo la instrucción en el curso preparatorio en su mayor parte han solicitado su correspondiente carta de ciudadanía, tendiendo a la nacionalización de la enseñanza en lo referente al personal... (EL TRIBUNO, 1911, s/p).

de Instrucción Pública.

${ }^{19}$ Ese año se incluyen solicitudes de ingreso a los cursos temporarios de escuelas de la Jewish. 
Las modificaciones plasmadas en la reforma constitucional, que alteraban la educación especial sostenida hasta la fecha, exceptuaban a la Escuela Normal Rural. Su tarea resultaba central al constituirse en el espacio clave que formaba al maestro que "surja de las colonias ${ }^{20}$ y que actuaría colaborando en la transformación del paisaje productivo de la provincia “...pues tanto en el aula como en sus relaciones con los vecindario son gallardos pregoneros de nuestro espíritu nacional...” (MEMORIA DE LA DIRECCIÓN GENERAL DE ENSEÑANZA, 1911 p.184).

Los cambios que experimentó esta institución estaban vinculados a su funcionamiento interno, producto de la experiencia práctica acumulada por los diferentes actores institucionales en esta etapa. Una de estas modificaciones se institucionalizó por medio de la resolución de la Dirección General de Enseñanza del 5 de abril 1909 que reorganizó el personal y modificó el reglamento, separando las funciones del jefe de cultivos de las de vice director. En esa oportunidad el Director quedó como responsable de las funciones de vice y las cátedras correspondientes. El Jefe de Cultivos debía ser un ingeniero agrónomo y tenía bajo su responsabilidad la Sección Ganadería con las cátedras respectivas. $\mathrm{Al}$ año siguiente, en octubre de 1910, un conflicto con un agrónomo responsable de varias materias específicas motivó la intervención del Inspector General que resolvió "refundir en un solo cargo que llevara denominación de Jefe de Cultivos y encargado de la Sección Ganadería con las cátedras correspondientes”. El Vicedirector debía ser un profesor normal encargado de la crítica pedagógica y de la regencia de la Escuela de Aplicación y se suprimieron los celadores. Dificultades como el tiempo de trabajo de los alumnos-maestros en las secciones, el escaso rendimiento de los cultivos y las permanentes dificultades en la organización de las secciones productivas impulsaron estos cambios.

\footnotetext{
20 Se otorgaron diez becas de la escuela normal de maestros rurales a jóvenes israelitas argentinos, quienes contraerán la obligación de servir un mínimo de tres años en escuelas judías. Se requirió al directorio de la Jewish Colonization que en toda escuela de su dependencia que tenga dos o más maestros, uno debía ser argentino y dictaría Idioma Nacional, Historia y Geografía, Instrucción Moral y Agricultura.
} 
En cuanto al plan de estudios, en 1910, se implementaron algunas modificaciones que habían sido ensayados el año anterior. Entre las reformas se incluyó la reducción del área de enseñanza agropecuaria a 125 hectáreas y la dependencia del Jefe de Cultivos del Director, medidas que fueron recién formalizadas en 1912. Varios enfrentamientos entre el director y los agrónomos responsables de las secciones productivas, con amplia repercusión en la prensa local, originaron la intervención e investigación sumaria en la escuela, que derivó en el desplazamiento del director Amadeo Autcher, que había estado a cargo desde fines de 1908. Hacia 1911 ya habían egresado 60 maestros normales rurales que se desempeñaban en las escuelas elementales con anexo agropecuario. Se registraba un aumento progresivo en la inscripción que, para ese año, comprendía 65 alumnos becados. 
Tabla 3. Plan de estudio de la escuela Juan Bautista Alberdi (1910) ${ }^{1}$

\begin{tabular}{|c|c|c|c|}
\hline \multirow{2}{*}{ MATERIAS } & \multicolumn{3}{|c|}{ AÑO } \\
\hline & $1^{0}$ & $2^{0}$ & $3^{0}$ \\
\hline Pedagogía & $\checkmark$ & $\checkmark$ & $\checkmark$ \\
\hline Aritmética & $\checkmark$ & $\checkmark$ & - \\
\hline Castellano & $\checkmark$ & $\checkmark$ & $\checkmark$ \\
\hline Historia & $\checkmark$ & $\checkmark$ & $\checkmark$ \\
\hline Geografía & $\checkmark$ & $\checkmark$ & $\checkmark$ \\
\hline Moral y Urbanidad & $\checkmark$ & - & - \\
\hline Agricultura general & $\checkmark$ & $\checkmark$ & - \\
\hline Ciencias Naturales & $\checkmark$ & - & - \\
\hline Dibujo Lineal & $\checkmark$ & - & - \\
\hline $\begin{array}{l}\text { Observaciones de la enseñanza en la Escuela de Aplicación anexa a la } \\
\text { Normal }\end{array}$ & $\checkmark$ & - & - \\
\hline Geometría Plana y Dibujo Lineal & - & $\checkmark$ & - \\
\hline Industrias Rurales & - & $\checkmark$ & $\checkmark$ \\
\hline Zootecnia general & - & $\checkmark$ & - \\
\hline Horticultura y Arboricultura & - & $\checkmark$ & - \\
\hline Nociones generales de Física & - & $\checkmark$ & - \\
\hline $\begin{array}{l}\text { Práctica de la enseñanza en la Escuela de Aplicación y critica } \\
\text { pedagógica }\end{array}$ & - & $\checkmark$ & $\checkmark$ \\
\hline Geometría del espacio Nociones de topografía y dibujo natural & - & - & $\checkmark$ \\
\hline Nociones de Química General & - & - & $\checkmark$ \\
\hline Medicina Veterinaria & - & - & $\checkmark$ \\
\hline Instrucción Cívica & - & - & $\checkmark$ \\
\hline Agricultura especial & - & - & $\checkmark$ \\
\hline Zootecnia & - & - & $\checkmark$ \\
\hline Contabilidad & - & - & $\checkmark$ \\
\hline
\end{tabular}

Fuente: Archivo General Entre Ríos. Serie Educación, Escuela Alberdi Caja $\mathrm{N}^{\circ} 2$.

Para los Cursos Temporarios, el 5 de febrero de 1911, se estableció una mayor formalización al ordenarse los programas en dos años consecutivos y reducirse los contenidos de acuerdo a las necesidades de la escuela rural -que tenía una duración menor a las escuelas elementales-. También se aumentó la cantidad de tiempo destinada a la práctica que incluía dos horas para clases modelos dictadas por los maestros y dos horas para las prácticas agropecuarias. La centralidad que tenía el espacio de las prácticas se basaba que la necesidad de familiarizar a los maestros en un corto tiempo para el desarrollo de las materias especiales y para vincular "...la Historia, la Geografía, la Instrucción Moral y Cívica y la Música con los cantos patrióticos para imprimir a la escuela 
el sello característico de la nacionalidad...” (MEMORIA DE LA DIRECCIÓN GENERAL DE ENSEÑANZA, 1911 p. 37). Las materias, se organizaban en un primer término que comprendía: Pedagogía, Aritmética, Historia, Geografía, Instrucción Cívica y Moral, Anatomía, Fisiología e Higiene, Geometría y Dibujo, Agricultura General, Zootécnica, Botánica y Música. En el segundo término se cursaban las mismas materias, excepto Botánica, pero con un grado de mayor complejidad, agregándose Agricultura Especial y Clasificación Zoológica.

Otra disposición para resolver el problema de la falta de titulación de los maestros fue la resolución de diciembre de 1912 que otorgaba el título de maestro rural a quienes comprobaran tener 10 años de buenos servicios. Tanto los Cursos Temporarios como la formación brindada en la Normal Rural se convertían en espacios claves para la apropiación de los saberes del oficio de maestro, las principales técnicas modernas para el fomento de la agricultura y ganadería, así como también la adhesión e incorporación a los rasgos sobresalientes del discurso nacionalista. La presencia de maestros extranjeros en estos cursos significaba que los mismos egresarían:

(...) no tan solo con el titulo de maestros argentinos sino con la carta de ciudadanía argentina cuya obtención la propia escuela normal le facilita $\mathrm{y}$ con sentimientos $\mathrm{y}$ tendencias nacionales que van fervorosamente a infiltrar a las poblaciones extranjeras de donde proceden, convirtiéndose así en el mejor factor de nacionalización de aquellos pobladores... (MEMORIA DIRECCIÓN GENERAL DE ENSEÑANZA 1913, p 55).

Los Cursos Temporarios de 1913-1914 incluyeron enseñanzas especiales: trabajo manual educativo para ambos sexos; labores a mano, economía domestica y jardinería para mujeres. Una de las modificaciones más sobresalientes fue el dictado para mujeres que, exceptuando el primer año de su implementación, sólo habían podido acceder a las mesas examinadoras. Las autoridades educativas evaluaban que, al no cursar la preparación básica que se dictaba en la Escuela "Alberdi", se generaba una desventaja en los exámenes. 
Por este motivo, se restablecieron los Cursos Temporarios para mujeres en la ciudad de Paraná y Concepción del Uruguay. El plan de estudios estaba distribuido en dos términos donde se dictaban las materias de las escuelas rurales, con mayor extensión e intensidad, y las materias especiales de carácter práctico, adaptadas a la mujer y al varón. Se establecía una importante carga horaria de cursado hasta el día sábado, destinándose la mañana para las materias teóricas y la tarde para las prácticas. Los domingos se dictaban conferencias sobre Medicina y profilaxis de enfermedades comunes en las escuelas. En 1914 se promovió la incorporación de mujeres al curso normal registrándose cuatro solicitudes de ingreso de alumnas externas, ya que la escasa infraestructura impedía su permanencia en la institución.

\section{CONCLUSIONES}

Se puede concluir que los Cursos Temporarios lograron cierta profesionalización de la tarea docente en el medio rural, posibilitaron el mejoramiento de las estadísticas provinciales en lo relativo a la cantidad de maestros titulados de las escuelas fiscales y un cierto control sobre el ejercicio del magisterio en las escuelas particulares. Con una preparación mínima se garantizaba que el personal docente reconociera los principales lineamientos de la enseñanza elemental de los contenidos más directamente vinculados a la nacionalización -geografía, historia, idioma nacional- y los conocimientos agropecuarios. Finalmente, se consolidaron dentro de la propuesta que brindaba la Escuela Normal Rural. La presencia femenina en la realización de los Cursos Temporarios y las mesas examinadoras para la obtención del título de competencia, mostraba un panorama más complejo del ejercicio del magisterio en el campo. El restablecimiento del dictado de los mismos para la población femenina, casi al final del período, permite conjeturar que la demanda de su reposición se produjo porque constituía una posibilidad laboral para las mujeres en el medio rural. 
Por su parte, la creación de una Escuela Normal Rural constituía un proyecto que circulaba desde fines del siglo XIX, impulsado por los funcionarios e intelectuales del campo pedagógico, preocupados por resolver la capacitación de los maestros rurales. Sin embargo, la fundación de la Escuela logró concretarse cuando se integró dentro de un conjunto de creaciones institucionales con propósitos de preparar personal idóneo en las actividades agropecuarias para desenvolverse en las zonas de colonización agrícola. La observación de la planta de personal, el plan de estudios y el reglamento interno, con las modificaciones que registró, mostró la progresiva consolidación que tuvo esta institución en relación a las escuelas agropecuarias creadas casi en simultáneo. La importante presencia que tenían los profesores normales en la dirección y dictado de las materias, la jerarquización de la profesión docente rural que ofrecía esta propuesta puede explicar, en parte, su permanencia a pesar de las fuertes críticas y resistencia que genero dentro y fuera del oficialismo.

Aunque la reforma de 1909 alteró el apoyo legislativo y financiero a la educación agropecuaria, la Escuela Normal como los Cursos Temporarios permanecieron y expandieron su acción en las comunidades inmigrantes, especialmente rusas y judías. Puede concluirse que la política educativa impulsada desde el Estado provincial para la formación del magisterio rural funcionó como una estrategia de mediación cultural con las diferentes comunidades inmigrantes. Los contenidos y materias que tendían a la nacionalización actuaron en conjunto con los conocimientos y prácticas agropecuarias, resultando una manera original de asegurar cierta efectividad en la intervención de los maestros, concebidos como las principales o únicas referencias de la estatalidad entre comunidades étnicas diversas que poblaban el territorio. 


\section{REFERENCIAS}

GUIRRE ROJAS, Carlos Antonio.La historia regional en la perspectiva de la corriente francesa de los Annales. Historia y Memoria. 2015, n.11, pp.273297

ALLIAUD, Andrea. Los maestros y su historia: los orígenes del magisterio argentino. Buenos Aires: Centro Editor de América Latina, 1993.

ARCHIVO GENERAL DE ENTRE RÍOS, Fondo Educación Serie Escuela “Juan Bautista Alberdi” Caja No 1, 2, 3,4, 5, 6 y 7 (1904 a 1914).

ARCHIVO GENERAL DE ENTRE RÍOS Fondo Educación. Serie XI Dpto. Paraná Cajas $\mathrm{N}^{0} 5,6,7,8,9,10,11$ 12, 13, 14.

ASCOLANI, Adrián. Las Escuelas Normales Rurales en Argentina. Una transición entre las aspiraciones de la cultura letrada el imaginario de cambio socioeconómico agrario (1900- 1946). En WERLE, Flavia (comp.), Educação Rural em Perspectiva Internacional. Instituições, práticas e formação do professor. Ijuí, Brasil: Editora UNIJUI, 2007. p. 373-424.

ASCOLANI, Adrián, Los balances de Historia de la Educación en Brasil: optimismo e incertidumbre de una producción expansiva. Comentarios en perspectiva comparada con Argentina y México. En NEPOMUCENO, Maria \& TIBALI, (org.), Educação e seus Sujeitos na História, Belo Horizonte, SBHE/Argvmentvm Editora, 2007, pp. 121-148.

BOHOSLAVSKY, Ernesto y SOPRANO, Germán. Un Estado con rostro humano. Funcionarios e instituciones estatales en Argentina (de 1880 a la actualidad), Universidad Nacional de General Sarmiento y Prometeo Libros, Buenos Aires, 2010.

CARLI, Sandra. Modernidad, diversidad cultural y democracia en la historia educativa entrerriana (1883-1930). En PUIGGROS, Adriana. (Dir.), La educación en las provincias y territorios nacionales (1885-1945). Historia de la educación en la Argentina IV. Buenos Aires: Galerna, 1993. p.185-237.

CIVERA, Alicia. Alcances y retos de la historiografía sobre la escuela de los campos en América latina (siglos XIX y XX) en Cuadernos de Historia, 34, Universidad de Chile, 2011.

CIAN, Janet. Orígenes de las escuelas agropecuarias en la provincia de Entre Ríos, Argentina 1896-1910. En Mundo Agrario No 42, Vol. 19 diciembre 2018. 
Universidad Nacional de La Plata. Disponible en https://www.mundoagrario.unlp.edu.ar/article/view/MAeo93

DUPUY, Víctor. Renuncia del Inspector de Escuelas de Entre Ríos. Prof. Víctor Dupuy. Su aceptación. Exposición. Rectificación. Paraná, 1913.

FIORUCCI, Flavia. Maestros para el sistema de educación pública. La fundación de escuelas normales en Argentina (1890-1930) en Revista Mexicana de Historia de la Educación, vol. II, núm. 3, 2014, pp. 25-45, 25 vol. II, núm. 3, 2014, p. 25-45. Recuperado en:http://ri.conicet.gov.ar/bitstream/handle/11336/35527/CONICET Digital Nro.08b2aae6-1c72-4ef2-9c32-1a0596fb631b A.pdf?sequence $=2$ \&isAllowed $=\mathrm{y}$

GONÇALVES VIDAL, Diana, O exercício disciplinado do olhar: livros, lecturas e práticas de formação docente no Instituto de Educação do Distrito Federal (1932-1937). Bragança Paulista: editora da Universidade São Francisco, 2001.

GUTIÉRREZ, Talia. Educación, agro y sociedad. Políticas educativas agrarias en la región pampeana 1897-1955. Buenos Aires: Editorial Universidad Nacional de Quilmes, 2007.

GUTIÉRREZ, Talia. Políticas de orientación agrícola y pedagogía normalista. Entre Ríos, Argentina, 1900-1920. Perfiles Educativos, 29(117), 85-110. Universidad Nacional autónoma de México, 2007. Recuperado en http://www.redalyc.org/articulo.oa?id=13211705

DIKER, Gabriela y TERIGI, Flavia. Formación de maestros y profesores: hoja de ruta. Buenos Aires: Paidos, 1997.

EL TRIBUNO, Ministerio de Gobierno 8 de abril de 1904 s/p

EL TRIBUNO, Los Cursos Temporarios, 18 de diciembre de 1910 s/p

ENTRE RÍOS Boletín de Educación, septiembre-diciembre, 1905.

ENTRE RÍOS Cámara de Senadores, Diario de sesiones, $\mathbf{1 3}^{\circ}$ sesión ordinaria, 25 de julio de 1903, 25 de julio p 38- 39.

ENTRE RÍOS Constitución de la Provincia de Entre Ríos, 1903.

ENTRE RÍOS Constitución de la Provincia de Entre Ríos, 1909.

ENTRE RÍOS Memoria presentada a la honorable legislatura de la provincia por el ministro de gobierno Ing. Alberto Mendez Casariego 
Tomo I, 1903-1904. Buenos Aires: Compañía Sud Americana de Billetes de Banco, 1904.

ENTRE RÍOS Mensajes de Gobernador Salvador Macia 1895 y 1896. ENTRE RÍOS Ley de Presupuesto General de la administración 1905. ENTRE RÍOS Memoria de la Dirección General de Escuelas año 1911. Buenos Aires: Mentruyt, 1912.

ENTRE RÍOS Memoria de la Dirección General de Escuelas año 1912. Buenos Aires: Mentruyt, 1913.

ENTRE RÍOS, Recopilación de Legislación de Educación Pública 1904 $(\mathrm{s} / \mathrm{l}, \mathrm{s} / \mathrm{n})$.

LIONETI, Lucia. La misión política de la escuela pública. Formar a los ciudadanos de la república 1870-1916. Buenos Aires: Miño y Dávila, 2007.

LÓPEZ, María del Pilar. La educación de rusos judíos y alemanes del Volga en Entre Ríos. Conflictos político-pedagógicos (1880-1910). En Ascolani, Adrian. (comp.), La educación en Argentina. Estudios de historia (pp 71-83). Rosario: Ediciones del Arca, 1999.

PEREYRA, Elsa. El Estado y la Administración Pública nacional en perspectiva histórica. Análisis crítico de la producción académica sobre el período 19301976. Revista Polhis, Vol. 5, No 9, Primer semestre 2012, p. 92-112.

SCHUELER, Alessandra y SOUTHWELL, Myriam. Formación del Estado Nacional y Constitución de los cuerpos docentes. Profesionalización del magisterio en Brasil y Argentina en perspectiva comparada (1820-200o. En Ascolani, Adrian y Vidal, Diana coord. Reformas educativas en Brasil y en Argentina. Ensayos de historia comparada de la educación. Buenos Aires, Biblos, 2011.

SOLIS, CARNICER, María del Mar, La historia regional en la Argentina. Sus aportes y desafíos para el futuro. Folia Histórica del Nordeste, Resistencia, diciembre 2015, pp. 165-168.

TEDESCO, Juan Carlos. Educación y sociedad en la Argentina (18801945). Buenos Aires: Ediciones Sol, 1986.

TERAN, Oscar. Historia de las ideas en Argentina. Diez lecciones iniciales, 1810-1980. Buenos Aires: Siglo XXI, 2008.

PUIGgROS, Adriana. Historia de la Educación Argentina. T 1. Buenos 
Aires: Editorial Galerna, 1990.

YANNOULAS, Silvia. Educar ¿una profesión de mujeres? Buenos Aires, Kapelusz, 1996.

WERLE, Flavia. Formação de professores para oensino de primeiras letras na zona rural. Brasil, final do século xix História da educação, asphe/fae/ufpel, pelotas, v. 12, n. 24, p., jan/abr 2008. p.121-153. Disponible en: http//fae.ufpel.edu.br/asphe.

ZUBIAUR, José. La escuela Primaria en Francia. Buenos Aires: Taller Tipográfico de La Penitenciaria, 1891.

JANET PRISCILA CIAN. es Prof. en Ciencias de la Educación y Doctoranda en Ciencias Sociales por la Universidad Nacional de Entre Ríos (UNER, Argentina). Becaria Doctoral del Consejo Nacional de Investigaciones Científicas y Técnicas (CONICET) con sede en el Instituto Rosario de Investigaciones en Ciencias de la Educación, Universidad Nacional de Rosario (UNR). Se desempeña como docente e investigadora en la Facultad de Ciencias de la Educación (UNER) y en la Facultad de Humanidades, Artes y Ciencias Sociales (UADER).

E-mail: janetcian@hotmail.com

(B) http://orcid.org/0000-0001-5747-4301

Recebido em: 28 de março de 2019

Aprovado em: 28 de junho de 2019

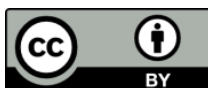

Revista História da Educação - RHE

Associação Sul-Rio-Grandense de Pesquisadores em História da Educação - Asphe Artigo de acesso aberto distribuído nos termos de licença Creative Commons. 